\title{
A Double-Blind, Placebo Controlled Trial, Evaluating the Effects of a Proprietary Blend of Plant Adaptogens on Sexual Satisfaction and Sexual Response in Adult Males
}

\author{
Gerry Lane*
}

\author{
Metabolic and Genetic Research Institute, Largo, Florida, USA
}

\begin{abstract}
Introduction: Adaptogens are plant substances that offer resistance of the organism to stressors. The human genome is able to utilize these plant derived substance to increase cellular defense in-vivo. Previous studies which validated adaptogens benefit in athletic training and performance also demonstrated subjective reports of improved male sexual performance. It is the objective of this trial is to isolate and report the human male sexual response to a proprietary blend of adaptogens, androgenic herbs combined and select amino acids (Admax $® /$ Potenter $®$ ) and compare it to placebo, over a 14 day period when compared to placebo.

Methods: Subjects were randomized into two cohorts to compare study compound $(n=42)$ with placebo $(n=21)$. Exclusion criteria eliminated individuals with organic impotence and organic heart disease as well as those unable to give informed consent or unable to adhere to study protocol. Participants completed the International Index of Erectile Function (IIEF) prior to dosing and instructed to attempt sexual intercourse a minimum of twice per week during the study period. They also were instructed to complete a sexual satisfaction diary, which became part of the source documents upon completion of the trial. Each participant was provided 50 capsules of either study compound or placebo (8 capsules per day with 2 additional capsules). Participants completed the IIEF upon completion of the study as well.

Results: 63 healthy adult males (age range 30-58, mean $=42.4, \pm 3$ ), were consented and enrolled in a placebo controlled, double blind, study of the male sexual experience and satisfaction in response to a proprietary blend of adaptogens (Admax/Potenter ${ }^{\circledR}$ ). All participants completed the trial and submitted both pre \& post self assessment utilizing the International Index of Erectile Function (IIEF). Participants on study compound $(n=42)$ improved in all 5 measured parameters, compared to no change in the placebo group $(n=21)$. All data was validated utilizing 2-tailed Student - T. Erectile function improved from 11.5 to 22.7 ( $p \leq 0.04$ ) compared to placebo (decreased from11.7 to 10.8). Intercourse satisfaction improved from 5.6 to 11.1 ( $p \leq 0.05$ ) compared to placebo (decreased from 5.5 to 5.2 ). Orgasmic function improved from 4.6 to 8.7 ( $p \leq 0.05$ ) compared to placebo (increased from 4.7 to 4.8 , not statistically significant). Sexual desire improved from 5.4 to 8.6 ( $p \leq 0.1)$ compared to placebo (decreased from 5.3 to 5.2 ). Overall sexual satisfaction improved from 3.1 to 9.1 ( $p \leq 0.01$ ) compared to placebo (decreased from 3.3 to 2.9 ).

Discussion: This data demonstrates that the male sexual experience is markedly enhanced with the utilization of this adaptogenic compound (Admax $\AA / P o t e n t e r \AA)$, when taken 3-6 hours prior to anticipation of sexual intercourse. Subjectively, the data would also indicate that the effect is relatively short acting (lasting 6-24 hours) and moderate onset of action (taken 3-6 hours prior to event). Pharmacokinetic studies were not performed. There were no side effects reported by any of the participants as is consistent with its use in previous clinical trials. All early indications would suggests, that this proprietary blend of adaptogens is an excellent supplement to increase the male sexual experience and can safely be used up to 14 days without demonstrated side effects.
\end{abstract}

Keywords: Adaptogens, sexual satisfaction, male sexual response, Admax/Potenter®.

\section{INTRODUCTION}

Adaptogens, (also known as phytoadaptogens) are defined as substances of plant origin that are able to increase a nonspecific resistance of the organism to stress factors and thereby promote its adaptation to stressful external conditions [1]. The human body has the ability to convert these plant derived substances to be utilized by our metabolic pathways to act in a similar cellular defense mechanism [2]. Biologically we are constantly under a condition of stress and certain amounts of stressors are not only normal but necessary. In fact, without stress, growth and development would be impaired. However, there are

*Address correspondence to this author at the Metabolic and Genetic Research Institute, Largo, Florida, USA; Tel: 614-946-3588;

E-mail: ifmresearch@aol.com many situations where stress is greater than the norm and if left unchecked, it can cause illness, injury or death. In everyday language, the term "stress" usually means an adverse reaction to some sort of unpleasant situation or danger. These stressors adversely affect physiologic sexual performance and function as well as psychological processes involved in sexual pleasure. Impairment of blood flow to the sexual organs combined with decreased erectile function negatively impacts the male sexual milieu.

It is postulated that a compound, such as found in adaptogens, that has improved the body's response to stressors as well as improved blood flow [3], will also improve the male sexual response. In previous clinical trials, utilizing the 4 primary adaptogens (Leuzea carthamoides, Rhodiola rosea, Eleutherococcus senticosus \& Schizandra chinensis) in the performance 
of athletes, there were also subjective reports of improved male sexual performance and overall satisfaction with the sexual experience. The effects on sexual performance of Tribulus terrestris extract [4], Lepidium meyenii extract [5] and Eurycoma longifolia extract [6] have been previously studied and reported. In a study with mice, $T$. terrestris was shown to enhance mounting activity and erection better than testosterone cypionate [4]. The proerectile aphrodisiac properties were concluded to likely be due to the release of nitric oxide [7] from the nerve endings innervating the corpus cavernosum penis. Cormio [8], et al. demonstrated that L-Citrulline supplementation, which stimulates endothelial nitric oxide, can offer short term erectile hardness when compared to placebo. Likewise the role of L-arginine in the production and release of endothelial nitric oxide has been documented in the literature; however, it is only effective in individuals who have diminished nitric oxide due to cardiovascular disease [9]. The primary benefit of Larginine is its conversion in the liver via Cyto-P450-sC3 enzymatic action into Citrulline and nitric oxide [10].

\section{Objective}

It is the objective of this trial is to isolate and report the human male sexual response to this proprietary blend of adaptogens, androgenic herbs combined with select amino acids and compare it to placebo, over a 14 day period when compared to placebo.

\section{METHODS}

Enrollment in the study required that all subjects were male adults, between the age 30 and 60 and met the following inclusion criteria:

- Able to offer informed consent, legally and cognitively

- $\quad$ Medically stable and healthy

- In a stable, sexually active relationship

- Willing to participate in a structured research project and complete the required documents

- Willing to attempt sexual activity a minimum of twice a week for 2 consecutive weeks

The participants were excluded from the study for the following reasons:

- History of Pyeronie's disease
- $\quad$ History of ambiguous genitalia

- Unable to have sexual intercourse 2 times in the subsequent 14 days for any reason.

- Unable to understand informed consent (English language only)

- Unable to legally give consent

- Unable to medically participate or receive clearance from primary care physician

- Unable to complete the 14 day course of clinical trial

- $\quad$ Any negative response to inclusion criteria

- Prior history of adverse reaction to any component of the trial compound

- Current utilization of Digoxin/Digitoxin/Digitalis.

Following verification of enrollees and affirmation of medical clearance from their PCP, each participant received either 50 capsules of study compound or placebo, randomized into 2 cohorts. One group of 42 males received active compound and a second group of 21 received placebo. Both participant and research coordinator were blinded during both the randomization and participation phases of the study. Un-blinding occurred only after data was gathered. Participants completed the International Index of Erectile Function (IIEF) [11] [see APPENDIX, RC Rosen, et al.], prior to dosing and instructed to attempt sexual intercourse a minimum of twice per week during the study period. The survey is designed to evaluate 5 key elements of the male sexual experience: 1) Erectile function, 2) Satisfaction with intercourse, 3) Orgasmic Function, 4) Sexual desire and 5) Overall satisfaction with the sexual experience. They also were instructed to complete a sexual satisfaction diary, which became part of the source documents upon completion of the trial. All participants were asked to take 8 capsules, daily for seven days, approximately 3-6 hours prior to sexual activity. Data was collected for a total of 14 days to determine approximate duration of action. Participants completed the IIEF upon completion of the study as well.

All data was collected and the study was closed, at which time the study compounds were un-blinded and statistical analysis of results was initiated. 
Table 1: International Index of Erectile Function

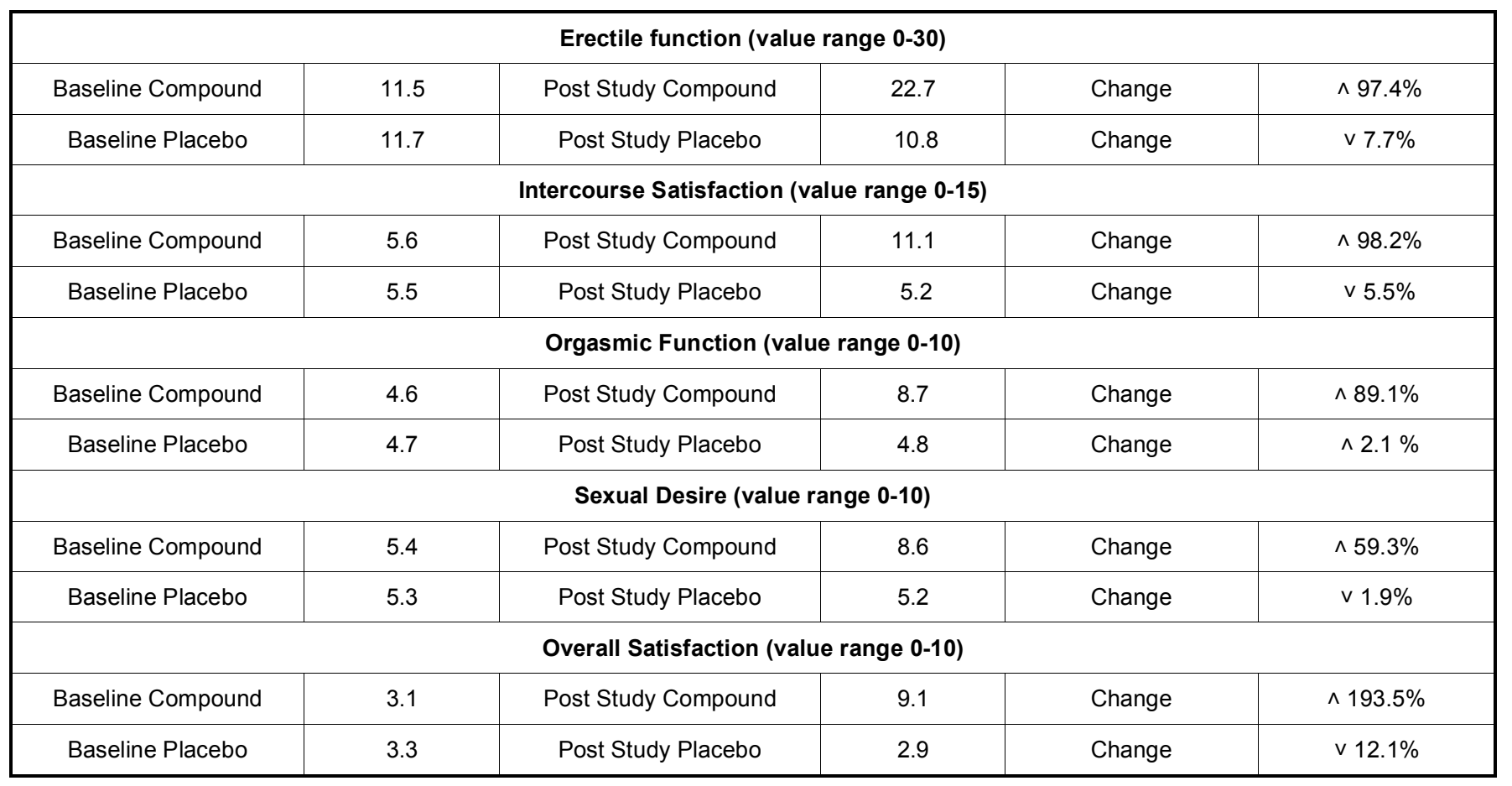

Note: Although, neither recruited nor excluded, there were no known transgender participants enrolled in the study.

\section{RESULTS \& STATISTICAL ANALYSIS}

POPULATION: 63 healthy adult males (age range $30-58$, mean $=42.4, \pm 3$ ), were consented and enrolled in a placebo controlled, double blind, study of the male sexual experience and satisfaction in response to a proprietary blend of adaptogens (Admax/Potenter® [12]). All participants completed the trial and submitted both pre \& post self assessment utilizing the International Index of Erectile Function (IIEF). Participants on study compound $(n=42)$ improved in all 5 measured parameters, compared to no change in the placebo group $(n=21)$. All data was validated utilizing 2tailed Student $-\mathrm{T}$. Erectile function improved from 11.5 to 22.7 ( $p \leq 0.04)$ compared to placebo (decreased from 11.7 to 10.8). Intercourse satisfaction improved from 5.6 to $11.1 \quad(p \leq 0.05)$ compared to placebo (decreased from 5.5 to 5.2). Orgasmic function improved from 4.6 to 8.7 ( $p \leq 0.05)$ compared to placebo (increased from 4.7 to 4.8 , not statistically significant). Sexual desire improved from 5.4 to $8.6 \quad(p \leq 0.1)$ compared to placebo (decreased from 5.3 to 5.2). Overall sexual satisfaction improved from 3.1 to 9.1 $(p \leq 0.01)$ compared to placebo (decreased from 3.3 to 2.9). Table 1 below illustrates these findings.

\section{DISCUSSION}

The participants in this scientific assessment where offered a proprietary blend of adaptogens, androgenic herbs and amino acids for the purpose of evaluating the male sexual response. Participants given placebo demonstrated no response in any of the 5 elements of the survey tool. In contrast, the recipients of the study compound had vastly favorable responses. All recipients of the study compound experienced greater ability to obtain and maintain an erection, they were far more capable of achieving orgasm and intercourse was a more satisfying event when compared to their baseline satisfaction ratings. Their overall satisfaction with the sexual experience demonstrated the most remarkable improvement with the relative value score increasing $193.5 \%$.

This data demonstrates that the male sexual experience is markedly enhanced with the utilization of this compound (Admax $® /$ Potenter $\circledast$ ), when taken 3-6 hours prior to anticipation of sexual intercourse. Subjectively, the data would also indicate that the effect is relatively short acting, lasting $6-12$ hours (mean $=7.5$ hrs \pm 2.5 hrs.) and moderate onset of action (taken 3-6 hours prior to event). Pharmacokinetic studies were not performed. There were no side effects reported by any of the participants as is consistent with its use in previous clinical trials. All early indications would suggests, that this proprietary blend of adaptogens is 
an excellent supplement to increase the male sexual experience and can safely be used up to 7 days without demonstrated side effects.

Adaptogens $=81.3 \mathrm{mg}$ (consisting of Leuzea carthamoides extract, Rhodiola rosea extract, Eleutherococcus senticosus extract, Schizandra chinensis extract)

\section{EACH CAPSULE CONTAINS}

500 mg L-Arginine

140 mg L- Citrulline

$6.3 \mathrm{mg}$ Leuzea carthamoides extract

$25 \mathrm{mg}$ Schizandra chinensis extract

$>25 \mathrm{mg}$ Rhodiola rosea extract
25 mg Eleutherococcus senticosus extract

$40 \mathrm{mg}$ Tribulus terrestris extract

40 mg Lepidium meyenii extract

$50 \mathrm{mg}$ Eurycoma longifolia extract

\section{ACKNOWLEGEMENT}

Dr. Lane is the Scientific Director of Metabolic and Genetic Research Institute: Largo, Florida. $\mathrm{He}$ is a graduate of Ohio University College of Osteopathic Medicine, Athens, Ohio and Past Associate Medical Director for Hilltop Research and Radiant Research, Columbus Ohio. Past Associate Professor of Medicine, Ohio University, College of Osteopathic Medicine, Dept of Family Medicine \& Geriatrics, Athens, Ohio

\section{APPENDIX: INTERNATIONAL INDEX OF ERECTILE FUNCTION}

The international index of erectile function (IIEF): a multidimensional scale for assessment of erectile dysfunction. Rosen RC, Riley A, Wagner G, Osterloh IH, Kirkpatrick J, Mishra A., Center for Sex and Marital Health, University of Medicine and Dentistry of New Jersey, Robert Wood Johnson Medical School, Piscataway 08854, USA.

Last 4 numbers of Soc. Sec. Date of Birth:

1. How often were you able to OBTAIN an erection during sexual activity (in the last 4 weeks)?

Answer

$0 . \quad$ No sexual activity

1. Never or almost never

2. Less than $1 / 2$ of the time

3. Sometimes, about $1 / 2$ of the time

4. Usually. More than $1 / 2$ of the time yet not every time

5. Always. Every time.

2. When you had an erection during sexual activity, was it SUFFICIENT (hard enough) for penetration (in the last 4 weeks)?

Answer
$0 . \quad$ No sexual activity
1. Never or almost never
2. Less than $1 / 2$ of the time
3. Sometimes, about $1 / 2$ of the time
4. Usually. More than $1 / 2$ of the time, yet not every time
5. Always. Every time.

3. When you attempted sexual intercourse, how did you ACTUALLY penetrate (in the last 4 weeks)?

\begin{tabular}{|c|c|c|}
\hline Answer & $\begin{array}{l}0 . \\
1 . \\
2 . \\
3 . \\
4 . \\
5 .\end{array}$ & $\begin{array}{l}\text { Did not attempt sexual intercourse } \\
\text { Never or almost never } \\
\text { Less than } 1 / 2 \text { of the time } \\
\text { Sometimes, about } 1 / 2 \text { of the time } \\
\text { Usually. More than } 1 / 2 \text { of the time, yet not every time } \\
\text { Always. Every time }\end{array}$ \\
\hline
\end{tabular}




\section{Subject ID:}

(last 4 \# of social security)

4. How often were you able to MAINTAIN penetration with intercourse (in the last 4 weeks)?

Answer

$0 . \quad$ Did not attempt sexual intercourse

1. Never or almost never.

2. Less than $1 / 2$ of the time

3. Sometimes. About $1 / 2$ of the time.

4. Usually. More than $1 / 2$ of the time, yet not every time

5. Always. Every time.

5. During sexual intercourse, how difficult was it to maintain an erection UNTIL COMPLETION of intercourse (in the last 4 weeks).

Answer

$0 . \quad$ Did not attempt intercourse

1. Extremely difficult. Was NEVER able to maintain an erection

2. Very difficult. Required significant effort or concentration

3. Difficult. Able to maintain with some focus

4. Slight difficulty. Only occasionally required some addition concentration

5. No difficulty. Always able to maintain erection until completion

6. How many time have you ATTEMPTED sexual intercourse, (in the last 4 weeks)

\begin{tabular}{|c|c|c|}
\hline Answer & $\begin{array}{l}0 . \\
1 . \\
2 . \\
3 . \\
4 . \\
5\end{array}$ & $\begin{array}{l}\text { Never. No attempts } \\
\text { Once or twice } \\
3 \text { to } 4 \text { attempts } \\
5-6 \text { attempts } \\
7-10 \text { attempts } \\
11 \text { attempts or areater }\end{array}$ \\
\hline
\end{tabular}

7. When you attempted sexual intercourse, how OFTEN was it satisfactory to you (in the last 4 weeks)

\begin{tabular}{|c|c|c|}
\hline Answer & $\begin{array}{l}0 . \\
1 . \\
2 . \\
3 . \\
4 . \\
5 .\end{array}$ & $\begin{array}{l}\text { Did not attempt sexual intercourse. } \\
\text { Never or almost never } \\
\text { Less than } 1 / 2 \text { of the time. } \\
\text { Sometimes. About } 1 / 2 \text { of the time } \\
\text { Usually. More than } 1 / 2 \text { of the time, yet not every time } \\
\text { Always. Every time. }\end{array}$ \\
\hline
\end{tabular}

8. How much have you ENJOYED sexual intercourse (in the last 4 weeks)?

Answer

$0 . \quad$ Did not have sexual intercourse

1. Never or almost never enjoyed intercourse

2. Enjoyed sexual intercourse a little

3. It was enjoyable, but I wish it had been better.

4. Highly enjoyable

5. Extremely enjoyable. I can't imagine any better.

9. When you had sexual stimulation or intercourse (in the last 4 weeks), HOW OFTEN did you ejaculate.

Answer

$0 . \quad$ Did not have sexual intercourse

1. Never or almost never ejaculated

2. Ejaculated less than $1 / 2$ of the time.

3. Ejaculated some of the time. About $1 / 2$ of the time.

4. Ejaculated often. More than $1 / 2$ of the time, yet not every time.

5. Ejaculated every time. 
10. When you had sexual stimulation or intercourse (in the last 4 weeks), HOW OFTEN DID YOU CLIMAX (have an orgasm)?
Answer
$0 . \quad$ Did not have sexual intercourse.
1. Never or almost never climaxed.
2. Climaxed less than $1 / 2$ of the time.
3. Climaxed some of the time. About $1 / 2$ of the time.
4. Climaxed often. More than $1 / 2$ of the time.
5. Climaxed ever time.

11. How often did you feel sexual DESIRE, (in the last 4 weeks)?

\begin{tabular}{|l|}
\hline Answer \\
\end{tabular}

$1 . \quad$ Never or almost never

2. Seldom. I rarely feel desire

3. Some times. I think about average

4. Frequently feel desire

5. Extremely often. I desire sex a lot.

12. How would you RATE YOUR LEVEL of sexual desire (in the last 4 weeks)?

\begin{tabular}{|l|}
\hline Answer \\
\end{tabular}
1. Very low
2. Below average
3. Moderate
4. Above average
5. Very high

13. Were you SATISFIED with your overall sex life (in the last 4 weeks)?

\begin{tabular}{|l|}
\hline Answer \\
\\
\end{tabular}
1. Extremely dissatisfied
2. Somewhat dissatisfied
3. Neutral. An equal mix of dissatisfaction and satisfaction
4. Somewhat satisfied
5. Extremely satisfied

14. How satisfied are you with your SEXUAL RELATIONSHIP with your partner (in the last 4 weeks)?

\begin{tabular}{|l|}
\hline Answer \\
\\
\end{tabular}

1. Extremely dissatisfied

2. Somewhat dissatisfied

3. Neutral. An equal mix of dissatisfaction and ratification

4. Somewhat satisfied

5. Extremely satisfied

15. How CONFIDENT were you that you would achieve and maintain an erection, to your satisfaction?

\begin{tabular}{|l|}
\hline Answer \\
\end{tabular}
1. Extremely low
2. Low
3. Moderate
4. High
5. Extremely high

\section{REFERENCES}

[1]

Panossian A, Wagner $\mathrm{H}$. Stimulating effect of adaptogens: an overview with particular reference to their efficacy following single dose administration. Phytother Res 2005; 19(10): 819-38.
[2] Anatoly Antoshechkin, M.D., PhD The Primary Adaptogens, Powerful Remedies of Prophylactic Medicine, Ceptima Publishing Co. Inc, ISBN 0-9700788-0-3.

[3] Antoshechkin AG, Olalde J, Margarici M, Muhammad A Salom A, Suarez J, Amendola F. Analysis of effects of the herbal preparation Circulat on gene expression levels in 
cultured human fibroblasts. Phytother Res 2007; 21(8): 777-89.

[4] Gauthaman K, Ganesan AP. The hormonal effects of Tribulus terrestris and its role in the management of male erectile dysfunction-an evaluation using primates, rabbit and rat. Phytomedicine 2008; 15(1-2): 44-54.

[5] Gonzales GF, Cordova A, Vega K, Chung A, Villena A, Gonez C, Castillo S. Effect of Lepidium meyenii (maca) on sexual desire and its absent relationship with serum testosterone levels in adult healthy men. Andrologia 2002; 34(6): 367-72.

http://dx.doi.org/10.1046/j.1439-0272.2002.00519.x

[6] Wahab NA, Mokhtar NM, Halim WN, Das S. The Effect of Eurycoma Longifolia Jack on Spermatogenesis in EstrogenTreated Rats. Clinics 2010; 65(1): 93-8. http://dx.doi.org/10.1590/S1807-59322010000100014

[7] Panossian A, Wikman G, Sarris J. Rosenroot (Rhodiola rosea): traditional use, chemical composition, pharmacology and clinical efficacy. Phytomedicine 2010; 17(7): 481-93. http://dx.doi.org/10.1016/j.phymed.2010.02.002
[8] Cormio L, De Siati M, Lorusso F, Selvaggio O, Mirabella L, Sanguedolce F, Carrieri G. Oral L-Citrulline supplementation improves erection hardness in men with mild erectile dysfunction 2011; 77(1): 119-22.

[9] Chen, Wollman, Chernichovsky, laina, Sofer and Matzkin. Effect of oral administration of high-dose nitric oxide donor Iarginine in men with organic erectile dysfunction: results of a double-blind, randomized, placebo-controlled study. BJU International 1999; 83: 269-273.

[10] Michael A. Marletta, Nitric Oxide Synthase: Aspects Concerning Structure and Catalysis. Cell 1994; 78: 927-930.

[11] The international index of erectile function (IIEF): a multidimensional scale for assessment of erectile dysfunction. Rosen RC, Riley A, Wagner G, Osterloh $\mathrm{IH}$ Kirkpatrick J, Mishra A., Center for Sex and Marital Health, University of Medicine and Dentistry of New Jersey, Robert Wood Johnson Medical School, Piscataway 08854, USA.

[12] Admax ${ }^{\mathrm{TM}} /$ Potender is a proprietary adaptogen from NuLab, Inc., Clearwater, FL, USA. 